\title{
A Systematic Study of the Dispersion of SWNTS in Organic Solvents
}

\author{
Qiaohuan Cheng \\ Technological University Dublin, qiaohuan.cheng@tudublin.ie \\ Sourabhi Debnath \\ Technological University Dublin, Sourabhi.debnath@tudublin.ie \\ Luke O'Neill \\ Technological University Dublin, Luke.oneill@tudublin.ie
}

See next page for additional authors

Follow this and additional works at: https://arrow.tudublin.ie/nanolart

Part of the Physics Commons

\section{Recommended Citation \\ Cheng, Q., Debnath, S., O'Neill, L., Hedderman, T., Gregan, E., Byrne, H.: A Systematic Study of the Dispersion of SWNTS in Organic Solvents. Journal of Physical Chemistry C, Vol. 114, (11), pp 4857-4863. 2010. doi:10.1021/jp911202d}

This Article is brought to you for free and open access by the NanoLab at ARROW@TU Dublin. It has been accepted for inclusion in Articles by an authorized administrator of ARROW@TU Dublin. For more information, please contact arrow.admin@tudublin.ie, aisling.coyne@tudublin.ie,gerard.connolly@tudublin.ie. Funder: Science Foundation Ireland Research Frontiers Program PHY037 2006. Raman instrumentation was funded under the framework of the INSPIRE programme, funded by the Irish Government's Programme for Research in Third Level Institutions, Cycle 4, National Development Plan 2007-2013, supported by the European Union Structural Fund.

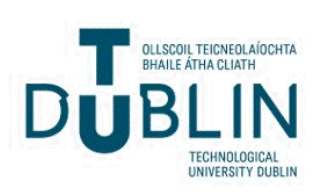


Authors

Qiaohuan Cheng, Sourabhi Debnath, Luke O'Neill, Theresa Hedderman, and Elizabeth Gregan

This article is available at ARROW@TU Dublin: https://arrow.tudublin.ie/nanolart/35 


\section{A systematic study of the dispersion of SWNTs in organic solvents}

Qiaohuan Cheng*, Sourabhi Debnath, Luke O'Neill, Theresa G. Hedderman, Elizabeth Gregan and Hugh J. Byrne

FOCAS Research Institute/School of Physics, Dublin Institute of Technology, Kevin Street, Dublin 8, Ireland

*Corresponding author. E-mail: qiaohuan.cheng@ dit.ie. Tel: +353 14027931.

\section{ABSTRACT}

Dispersions of as-produced HiPco single-walled carbon nanotubes (SWNTs) in a series of organic solvents were prepared by dilution with the aid of tip sonication. Mild centrifugation $(\sim 945 \mathrm{~g})$ was carried out to remove large bundles. Atomic force microscopy (AFM) studies revealed that the bundle size decreased as the dispersion was diluted. By measuring the UV-vis-NIR absorption before and after centrifugation as a function of the concentration, the dispersion limit of SWNTs in each solvent can be determined. Correlations between the dispersion limit and solvent solubility parameters, including the Hildebrand solubility parameter and three dimensional Hansen solubility parameters, are explored, demonstrating that SWNTs are easily dispersed in solvents with Hildebrand solubility parameter range from $\sim 22-24 \mathrm{MPa}^{1 / 2}$ and Hansen polarity component $\left(\delta_{p}\right) \sim 12-14 \mathrm{MPa}^{1 / 2}$. No clear correlation between dispersion limits and the dispersion force $\left(\delta_{D}\right)$ or hydrogen bonding force $\left(\delta_{H}\right)$ are evident. It is found, however, that the degree of dispersion depends critically on sample preparation conditions and in particular sonication time. Increased 
sonication times increase the amount of SWCNTs debundled and solubilised but do not appear to affect the dispersion limit. However, increased sonication also induces discernible changes to the SWNTs themselves and in itself influences their solubility, under which conditions no clear solubility parameters can be determined.

KEYWORDS: Single-walled carbon nanotubes, organic solvents, dispersion limit, sonication, Hildebrand solubility parameter, Hansen solubility parameter

\section{INTRODUCTION}

With novel structures and remarkable physical properties, single-walled carbon nanotubes (SWNTs) have been identified as promising nanomaterials for many applications [1-4]. However, due to the strong intertubular van der Waals interaction and chemical inertness [1], as prepared SWNT samples are difficult to separate and are relatively insoluble in either water or common organic solvents, a significant obstacle in the application of SWNTs. With no side wall modification or the introduction of a third component to the dispersion, the direct dispersion of as produced SWNTs in proper media, such as organic solvents, is a desirable method of purification and dispersion of SWNTs. Motivated by the good dispersibility of SWNTs reported in ortho-dichlorobenzene (o-DCB) and monochlorobenzene (MCB) [2, 3], a systematic study of the ability of chlorinated aromatic solvents, including MCB, o-DCB, meta-dichlorobenzene (m-DCB) and 1, 2, 4-trichlorobenzene (TCB), to disperse as-produced HiPco SWNTs was conducted [4], concluding that the phenyl ring in the solvent molecule is not the dominant factor in obtaining stable SWNTs dispersions. Subsequently, the dispersibility of SWNTs in chlorinated aromatic solvents and other solvents reported in literature, including dimethylformamide (DMF) [5, 6], 1,2-dichloroehtane (DCE) [7], chloroform [2], and toluene [8], in terms of optical extinction and absorption coefficients, was compared as a function of Hildebrand and Hansen solubility parameters [9]. 
The Hildebrand solubility parameter , which is defined as the square root of the cohesive energy density of a material $\left(\delta=\sqrt{\Delta H-R T / V_{m}}\right.$ ) [10], provides a numerical estimation of the intermolecular attractive force which must be overcome in the dispersion process [10]. The cohesive energy arises from three different interactions: (atomic) dispersion forces $\left({ }_{D}\right)$, (molecular) permanent dipole-permanent dipole forces $\left({ }_{P}\right)$, and (molecular) hydrogen bonding $(H)$, defined by the Hansen solubility parameters $[10,11]$. The correlation between the Hildebrand solubility parameter and Hansen solubility parameters for a chosen material is given by:

$$
{ }^{2}={ }_{D}^{2}+{ }_{P}^{2}+{ }_{H}^{2} \quad \text { Equation } 1
$$

The Hansen solubility parameters provide information regarding the origin of the interaction between the solvent and solute, similar Hildebrand and Hansen solubility parameters indicating the likelihood of miscibility. It may happen, however, that the solute and solvent have similar Hildebrand solubility parameters, dominated by different type of interactions, but are not miscible at all [10].

In suspensions of SWNTs, the relative amount of nanotubes can be determined by the optical absorption [2]. As the extinction/absorption coefficients are calculated from the absorbance of the suspension/solution, the values reflect the amount of tubes suspended/dispersed in the solution. Based on the extinction/absorption coefficients observed for the range of solvents, polar forces and hydrogen bonding have previously been found to be dominant compared to dispersion forces both for chlorinated aromatic solvents and other solvents, although the correlation appeared to differ for the two sets of solvents [9]. In order to more fully understand the behaviour of SWNTs in a chosen solvent, a further parameter, the dispersion limit or critical debundling concentration, should be considered. The dispersion limit is a measure of the ease of dispersion of SWNTs in a solvent. 
By measurement of the UV-visible-NIR absorbance before and after centrifugation as a function of concentration, Giordani et al. reported the method of calculating the dispersion limit in N-methyl-2pyrrolidone (NMP) [12]. This concentration corresponds to the point at which scattering from aggregates ceases to dominate the optical characteristics as a result of exfoliation or debundling upon dilution. The mass fraction of aggregates is described by

$$
\chi_{\text {agg }}=\frac{A_{\text {before }}-A_{a f t e r}}{A_{\text {before }}}
$$

where $A_{\text {before }}$ is the absorbance (chosen at 660nm) before centrifugation and $A_{\text {after }}$ is the absorbance at the same wavelength after centrifugation. The aggregate fraction was then plotted as a function of as-prepared concentration. The concentration at which the aggregation ceases to dominate the dispersion is considered to be the dispersion limit $\left(D_{L}\right)$ of SWNTs in the respective solvent. The same method was adopted in this work to monitor the dispersibility of SWNTs in the employed solvents.

In this work, the dispersion limit of as-produced HiPco SWNTs in a range of organic solvents was monitored and plotted as a function of the respective solvent Hildebrand and Hansen solubility parameters. Thirteen organic solvents were employed in this study. In order to further investigate the correlation between the dispersion limit and solvent Hildebrand and Hansen solubility parameters, based on the eight solvents investigated before, five additional solvents, 1, 2-dibromoethane (DBE), N-methyl-2-pyrrolidone (NMP), Nitromethane, acetonitrile and Dimethyl sulfoxide (DMSO), were added according to their solubility parameters.

AFM studies were employed to confirm that the centrifuged samples are dominated by isolated tubes and/or very small bundles at concentrations below the dispersion limit. Correlations between the dispersion limit and solvent solubility parameters are explored, demonstrating that SWNTs are easily dispersed in solvents with Hildebrand solubility parameter range from $\sim 22-24 \mathrm{MPa}^{1 / 2}$ and Hansen polarity component $\left({ }_{P}\right)$ around $\sim 12-14 \mathrm{MPa}^{1 / 2}$. Similar to the relationships previously determined for the extinction/absorption 
coefficients, the effect of dispersion force $\left({ }_{D}\right)$ is not evident. However, whereas the extinction was previously observed to be correlated with the hydrogen bonding parameter $\left({ }_{H}\right)$, no clear ${ }_{H}$ dependence of dispersion limit is observed here. Comparing to similar studies in literature, good agreement in terms of Hildebrand solubility parameters is seen here [13], but not in terms of Hansen solubility parameters. This disparity of the results reported here from those in literature is shown to be at least in part due to sonication conditions employed during sample preparation, which affect the degree of solubilisation but also the physical and/or chemical properties of the SWNTs themselves, bringing into question the validity of universal solubility parameters and suggesting the need for a systematic study of the sonication process and its dependence on solubility parameters.

\section{EXPERIMENTAL SECTION}

HiPco SWNT (Carbon Nanotechnologies Inc., batch number PO341) dispersions were produced by sonicating in each solvent using a high power ultrasonic tip processor (Ultrasonic processor VCX $750 \mathrm{~W}$ ) at $26 \%$ output for $20 \mathrm{~s}$, followed by serial dilution by 0.75 to produce a range of dispersions with concentrations from $0.21 \mathrm{mg} / \mathrm{ml}$ to $0.001 \mathrm{mg} / \mathrm{ml}$. The volume of each sample was $5 \mathrm{ml}$. All samples were then sonicated for an additional $100 \mathrm{~s}$ [4] to make sure each sample received the same sonication treatment. All the dispersions were allowed to settle for two days to minimize the effect if any of solvent density or viscosity and were subsequently centrifuged at $3000 \mathrm{rpm}(\sim 945 \mathrm{~g})$ (ECONOSPIN Sorvall Instruments) for 60 mins.

UV-vis-NIR absorption (Perkin-Elmer Lambda 900) measurements were performed both before and after centrifugation. Measurements were performed using an integrating sphere to minimize the contributions of scattering from residual aggregates and impurities to the measured extinction [9, 14]. 10-millimeter quartz cuvettes were used for all the measurements. UV-vis-NIR measurements were carried out on the whole sample before centrifuge (immediately before measurement all samples were vigorously shaken) but only the supernatant after centrifugation, so that the mass fraction of aggregates can be estimated (see Equation 
2). The absorption coefficient (AE) for SWNTs in each solvent was calculated from the slope of the absorbance obtained in the integrating sphere as a function of as prepared concentration [9].

Raman measurements were performed with a LabRAM HR800 Raman Microscope (Horiba Jobin Yvon) at a laser energy of $2.33 \mathrm{eV}(532 \mathrm{~nm})$ on dispersions drop cast onto quartz substrates. A x50 objective lens was used for all the measurements. Up to ten spectra were taken randomly for each sample. The intensities of the $\mathrm{D}$ band and $\mathrm{G}^{+}$were taken after base line correction and the ratios of $\mathrm{I}_{\mathrm{D}} / \mathrm{I}_{\mathrm{G}}^{+}$were calculated for all spectra and averaged.

The samples for AFM were prepared by drop casting the supernatant onto cleaned quartz substrates. AFM images were acquired on a MFP-3D-BIO ${ }^{\mathrm{TM}}$ Atomic Force Microscope (Asylum Research) in tapping mode.

In all cases, it is difficult to assess the actual final concentration of the sample. For the purposes of optimization of preparation techniques and comparison of solvents, consistent with previous works [4, 12], concentrations are quoted are those of as prepared samples.

\section{RESULTS AND DISCUSSION}

Figure 1 shows the concentration dependence of aggregation fraction (calculated with the absorption at 660nm) for SWNTs in MCB, TCB and DMF dispersions. In TCB, the aggregate fraction after centrifugation is reduced to $\sim 0.1$, indicating almost complete debundling at concentrations below $\sim 0.005$ $\mathrm{mg} / \mathrm{ml}$, and an absorbance which is almost unaffected by centrifugation. However, in the dispersions of $\mathrm{MCB}$, aggregates dominate the dispersion over the whole range of the concentration studied and are entirely removed by centrifugation. In this case, the dispersion limit of SWNTs in the corresponding solvent is considered to be $<0.001 \mathrm{mg} / \mathrm{ml}$. 
It should be noted that although the dispersion limit of SWNTs in DMF can be considered to be 0.022 $\mathrm{mg} / \mathrm{ml}$, the aggregation fraction below this limit is as high as 0.5 . Nevertheless, the exfoliation of the SWNTs bundles with dilution can be confirmed by AFM. Figure 2 shows the AFM images of the dispersion at $\sim 0.0375 \mathrm{mg} / \mathrm{ml}$ and $0.0067 \mathrm{mg} / \mathrm{ml}$ in DMF. It is clearly seen that the bundle size decreases with decreasing concentration until, below the dispersion limit, the SWNTs exist as isolated tubes or very small bundles $(2-3 \mathrm{~nm})$.

The dispersion limit of SWNTs in each solvent is listed in Table 1, together with the solvent solubility parameters of the respective solvent and the aggregation fraction below the dispersion limit. Figure 3 shows the location of the solvents employed in Hansen parameter space, the size of the spheres indicating the dispersion limit of SWNTs in the corresponding solvent. It is seen that the solvents employed occupy a wide range of polarity and hydrogen bonding values. However, it should be noted that the dispersion force values do not vary significantly over the range of solvents used, most values being located between 17 $\mathrm{MPa}^{1 / 2}$ to $20 \mathrm{MPa}^{1 / 2}$. Although several successful solvents appear in this range, it is not an appropriate parameter for defining a "good" solvent of SWNTs, as many solvents with similar values of ${ }_{D}$ are "poor" solvents.

For comparison, the absorption coefficients [9] and the dispersion limits of SWNTs in different solvents are plotted against the solvent solubility parameters in one plot. Figure 4 shows the absorption coefficient and dispersion limit of SWNTs in the solvents as a function of the corresponding Hildebrand solubility parameter. It is seen that the results of the absorption measurements match well with those of the dispersion limit. It was reported in a previous study of the absorption coefficient $v s$ Hildebrand solubility parameter that the chlorinated aromatic solvents and others can be fitted by two different trends [9]. When more solvents are included, however, it appears more appropriate to consider that both the absorption coefficient and dispersion limit are sharply peaked within a specific Hildebrand range, $\sim 22-24 \mathrm{MPa}^{1 / 2}$. This value agrees very well with the theoretical calculation of the Hildebrand solubility parameters, which was found to be $23 \mathrm{MPa}^{1 / 2}$ for SWNTs of $1 \mathrm{~nm}$ diameter [15]. Bergin et al. also reported the Hildebrand solubility 
parameter for HiPco SWNTs to be sharply peaked $21 \mathrm{MPa}^{1 / 2}$ [13], which is comparable to that indicated here. Solvents which have lower or higher Hildebrand solubility parameter have inferior dispersibility of bundled HiPco SWNTs, and notably acetonitrile, with a value of $\delta=24.2$, shows very poor dispersion of SWNTs. The narrow range of the distribution can be attributed to the general requirement that the Hildebrand parameter of the solvent match that of the solute. [Hildebrand, J. H.; Prausnitz, J. M.; Scott, R. L. Regular and Related Solutions, 1st ed,; Van Nostrand Reinhold Company: New York, 1970; p 228.]. There is considerable spread and indeed asymmetry in the results of Figure 4, however, suggesting that the Hildebrand parameter is not specific enough to describe the interaction between the solvent and SWNTs, and that the interaction may better be understood by examining correlations with the more specific Hansen parameters.

The correlations between absorption coefficient and dispersion limit and each of the three-dimensional Hansen solubility parameters are plotted and shown in Figure 5. Figure 5 (a) shows the distribution of the points as a function of ${ }_{D}$, and no clear correlation is observed between the absorption coefficients and dispersion limits. Although several successful solvents appear in the range $\sim 17-19 \mathrm{MPa}^{1 / 2}$, some solvents with ${ }_{D}$ within this range do not give good SWNT dispersions, and therefore it is deemed that this factor is not a adequate parameter to predict a "good" solvent for SWNTs. Figure 5 (b) indicates the correlation between the absorption coefficient and dispersion limit $v s$ the dipole- dipole force of the solvents $\left({ }_{P}\right)$. It is clearly seen that among the employed solvents, both the absorption coefficient and dispersion limit show a maximum in the ${ }_{\mathrm{P}}$ range from $\sim 12-14 \mathrm{MPa}^{1 / 2}$. This range is quite different with the value reported in the study of Bergin et al., in which a peak of dispersion limit was observed at ${ }_{P} \cdot 7.5 \mathrm{MPa}^{1 / 2}[13]$, although there does appear to be a local maximum at $\sim 6.5 \mathrm{MPa}^{1 / 2}[16]$. The distribution of the points as a function of the Hydrogen-bonding force $\left(_{\mathrm{H}}\right)$ is shown in Figure 5(c). The consistency of dispersion limit and absorption is less apparent compared to the plot of ${ }_{P}$. Although the solvents with ${ }_{H} \cdot 7 \mathrm{MPa}^{1 / 2}$ show better solubility of SWNTs compared to other solvents, no clear correlation between dispersion limit and absorption coefficient was observed for the employed solvents. 
Although the results of Figure 4 agree well with previous reports in terms of the optimum range of the Hildebrand parameter, there is significant discrepancy in terms of the optimal values of the Hansen parameters which should indicate the mechanisms underlying, and lead to a greater understanding of, the solubilisation process.

A critical consideration in the intercomparison of studies is the sample preparation conditions. It is clear that, in Figure 1, although DMF shows the highest dispersion limit of the employed solvents at $\sim 0.022$ $\mathrm{mg} / \mathrm{ml}$, the aggregation fraction below the dispersion limit is as high as 0.5 , indicating that only partial debundling has occurred. Indeed, the AFM image of figure 6 shows that, precentrifugation, the sample contains a significant number of bundles at a concentration of $\sim 0.003 \mathrm{mg} / \mathrm{ml}$. In Table 1 , it can be seen that the aggregation fractions below the dispersion limit of SWNTs in different solvents vary significantly and do not correlate with their ability to disperse SWNTs (dispersion limits). In order to explore this, a further two sets of SWNTs/DMF dispersions were sonicated for 4 mins and 6 mins respectively. The aggregation fractions for different sonication times were plotted as a function of prepared concentration, and compared to those presented in Figure 1, shown in Figure 7. It is clear that the degree of debundling below the dispersion limit is critically dependent on sonication time. The estimated dispersion limit however appears to be unaffected by the degree of sonication indicating that it may be determined by the solvent parameters rather than the sonication treatment.

Furthermore, it has been established that sonication not only helps to exfoliate the nanotube bundles, but also results in a cutting of the SWNTs or the introduction of defects on their side walls. Damage of the tubes can be monitored by the intensity of the D band of the Raman spectrum compared to that of the corresponding $\mathrm{G}^{+}$band, the $I_{D} / I_{G}{ }^{+}$ratio [17]. Raman spectra of SWNTs extracted from DMF dispersion, sonicated for different times, were taken and the ratio $I_{D} / I_{G}{ }^{+}$was calculated and plotted as a function of sonication time. As shown in Figure 8, the $I_{D} / I_{G}{ }^{+}$ratio increases significantly as the sonication time is increased, indicating that, although longer sonication time increases the debundling of SWNTs in solution, 
this is at the expense of alteration of the physical and/or chemical properties of the tubes themselves, and that damage to the SWNTS is evident even at lower sonication times. Thus, the very nature of the SWNTs and their solubility, is also dependent on the sonication time and the identification of a characteristic and unique set of solubility parameters is difficult. In the study of Bergin et al. [13], each sample was sonicated for 30mins with ice cooling, whereas in the work of Detriche et al. [16, 18], each sample was sonicated for 2 mins by a tip sonicator. In their study of HiPco nanotubes from Unidym, Bergin et al. identified optimal solubilisation for solvent Hildebrand parameters in the range $19<<24 \mathrm{MPa}^{1 / 2}$, with a maximum at 21 $\mathrm{MPa}^{1 / 2}$. The corresponding optimal ranges for the Hansen parameters were, ${ }_{D}: 17<{ }_{D}<19 \mathrm{MPa}^{1 / 2},{ }_{P}: 5<$ ${ }_{P}<14 \mathrm{MPa}^{1 / 2},_{\mathrm{H}}: 3<{ }_{H}<11 \mathrm{MPa}^{1 / 2}$, and the estimated parameters for SWNTs were ${ }_{D}=17.8 \mathrm{MPa}^{1 / 2},{ }_{P}=$ 7.5 $\mathrm{MPa}^{1 / 2}{ }_{H}=7.6 \mathrm{MPa}^{1 / 2}$. In the study of Detriche et al. of CVD SWNTs (with average diameter of $2 \mathrm{~nm}$ ), no details of sonication conditions are given, but the samples are additionally purified by concentrated $\mathrm{HCl}$. The optimal Hildebrand range is $20<<22 \mathrm{MPa}^{1 / 2}$, with Hansen parameters ${ }_{D}: 19<{ }_{D}<21 \mathrm{MPa}^{1 / 2},{ }_{P}: 4<$ ${ }_{P}<7 \mathrm{MPa}^{1 / 2},_{H}: 3<{ }_{H}<5 \mathrm{MPa}^{1 / 2}$, and the estimated parameters for SWNTs were: ${ }_{D}=19.4 \mathrm{MPa}^{1 / 2},{ }_{P}=$ 6.0 $\mathrm{MPa}^{1 / 2}{ }_{H}=4.5 \mathrm{MPa}^{1 / 2}$. A further study by Ham et al. utilized purified HiPco SWNTs soniocated for 20 hrs identified ${ }_{D}$ as the most important parameter with values in the range $17<{ }_{D}<18 \mathrm{MPa}^{1 / 2},{ }_{P}$ as having an upper limit of $14 \mathrm{MPa}^{1 / 2}$, and ${ }_{H}$ an upper limit of $12 \mathrm{MPa}^{1 / 2}$. There is thus considerable discrepancy between the studies already reported in literature and it is notable that the types of nanotubes and the preparation conditions very significantly between studies.

Sonication can also affect changes to the solvent characteristics, further complicating any correlation to solubility parameters. As shown in Figure 9, in the TCB dispersion, a foreign coating on the SWNTs is evident, as previously reported in $o$-dichlorobenzene (o-DCB)/SWNTs dispersions [19], although the sonication time in this study is only 2 mins compared to that of 3 mins- 60mins in reference [19]. It was reported that in $o$-DCB dispersions, sonication caused the decomposition and polymerization of $o$-DCB and the sonopolymer coated on the tubes was proposed to contribute to the stabilization of SWNT in $o$-DCB suspension [19]. Similarly, the observation of the sonopolymer in SWNTs/TCB samples might be 
responsible for the low aggregation fraction in TCB and the high dispersion limit of SWNTs in $o$-DCB. However, MCB, which has a similar structure to that of $o$-DCB and TCB, is a poor solvent for SWNTs and so correlations of such effects to solvent molecular structure are difficult.

\section{CONCLUSIONS}

Systematic studies of the interaction of SWNTs with organic solvents are critical to developing an understanding of solubilisation mechanisms and thus an optimisation of processing protocols. Good agreement with literature is demonstrated here in terms of Hildebrand parameters, but not in terms of the Hansen solubility parameters. It has been demonstrated that the degree of dispersion is critically dependent on sample preparation conditions, in particular sonication. Prolonged sonication clearly causes progressive physical and/or chemical modification of the SWNTs, however, and given that the material to be solubilised is ill defined, it is difficult to justify a universal or characteristic solubility parameter. The results indicate that further systematic investigation of the sonication process is merited in order to differentiate the solubilising effects from the results of physical and/or chemical modification of the samples themselves.

\section{ACKNOWLEDGMENT}

This project was funded under the Science Foundation Ireland Research Frontiers Program PHY037 2006. Raman instrumentation was funded under the framework of the INSPIRE programme, funded by the Irish Government's Programme for Research in Third Level Institutions, Cycle 4, National Development Plan 2007-2013, supported by the European Union Structural Fund. 


\section{REFERENCES}

(1) Rao, A.M., Chen, J.; Richter, E.; Schlecht, U.; Eklund, P.C.; Haddon, R.C.; Venkateswaran, U.D.; Kwon, Y.K.; Tomanek, D., Phys. Rev. Lett. 2001, 86, 3895.

(2) Bahr, J.L.; Mickelson, E.T.; Bronikowski, M.J.; Smalley, R.E.; Tour, J.M. Chem. Commun. 2001, 2 , 193.

(3) Kim, D.S.; Nepal, D.; Geckeler, K.E. Small. 2005, 1, 1117.

(4) Cheng, Q.; Debnath, S.; Gregan, E.; Byrne, H.J. Phys. Stat. Sol. (b). 2008, 245, 1947.

(5) Furtado, C. A.; Kim, U.J.; Gutierrez, H. R.; Pan, L.; Dickey, E. C.; Eklund, P. C. J. Am. Chem. Soc. 2004, 126, 6095.

(6) Landi, B.J.; Ruf, H.J.; Worman, J.J.; Raffaelle, R.P. J. Phys. Chem. B. 2004, 108, 17089.

(7) Kim, K. K.; Bae, D.J.; Yang, C.M.; An, K.H.; Lee, J.Y.; Lee, Y.H. J. Nanosci. Nanotechnol. 2005, 5, 1055.

(8) Hedderman, T. G.; S.M. Keogh; G. Chambers; Byrne, H. J. J. Phys. Chem B, 2004, 108, 18860.

(9) Cheng, Q.; Debnath, S.; Gregan, E.; Byrne, H. J. J. Phys. Chem C, 2008. 112, 20154.

(10) Burke, J. The Book and paper group annual, 1984, 3, 13.

(11) Hansen C. M., Hansen Solubility Parameters: A User's Handbook, CRC, 1999.

(12) Giordani, S.; Bergin, S.D.; Nicolosi, V.; Lebedkin, S.; Kappes, M.M.; Blau, W.J.; Coleman, J.N. J. Phys. Chem. B. 2006, 110, 15708. 
(13) Bergin, S.D.; Sun, Z.Y.; Rickard, D.; Streich, P.V.; Hamilton, J.P.; Coleman, J.N. Acs Nano, 2009, 3, 2340 .

(14) Priya, B.R.; Byrne, H.J. J. Phys. Chem. C. 2008, 112, 332.

(15) Maiti, A.; Wescott, J.; Kung P. Mol. Simulat. 2005, 31, 143.

(16) Detriche, S.; Zorzini, G.; Colomer, J.F.; Fonseca, A.; Nagy, J.B. J. Nanosci. Nanotechnol. 2008, 8, 6082.

(17) Giordani, S.; Bergin, S.; Nicolosi, V.; Lebedkin, S.; Blau, W.J.; Coleman, J.N. Phys. Stat. Sol. (b). 2006, 243, 3058.

(18) Detriche, S.; Nagy, J.B.; Mekhalif, Z.; Delhalle, J. J. Nanosci. Nanotechnol. 2009, 9, 6015.

(19) Niyogi, S.; Hamon, M.A.; Perea, D.E.; Kang, C.B.; Zhao, B.; Pal, S.K.; Wyant, A.E.; Itkis, M.E.; Haddon, R.C. J. Phys. Chem. B. 2003, 107, 8799.

(20) Abboud, J.L.M.; Notario, R. Pure Appl. Chem. 1999, 71, 645.

Table 1 The Hildebrand solubility parameter and Hansen solubility parameters of the solvents and the dispersion limits $\left(D_{L}\right)$ of SWNTs in different solvents, together with the aggregation fraction $\left(\chi_{\text {agg }}\right)$ below dispersion limit in each solvent and absorption coefficient (AE).

\begin{tabular}{|c|c|c|c|c|c|c|c|c|}
\hline Name & $\begin{array}{c}\text { Molecular } \\
\text { formula }\end{array}$ & $\begin{array}{c}\delta_{\mathrm{D}}^{[11]} \\
\left(\mathrm{MPa}^{1 / 2}\right)\end{array}$ & $\begin{array}{c}\delta_{\mathrm{P}}^{[1]]} \\
\left(\mathrm{MPa}^{1 / 2}\right)\end{array}$ & $\begin{array}{c}\delta_{\mathrm{H}}^{[1]]} \\
\left(\mathrm{MPa}^{1 / 2}\right)\end{array}$ & $\begin{array}{c}\delta^{[20]} \\
\left(\mathrm{MPa}^{1 / 2}\right)\end{array}$ & $\begin{array}{c}D_{L} \\
(\mathrm{mg} / \mathrm{ml})\end{array}$ & $\begin{array}{c}\chi_{\text {agg }} \\
\text { below } D_{L}\end{array}$ & $\begin{array}{c}\mathrm{AE} \\
\left(\mathrm{mlmg}-{ }^{1} \mathrm{~m}^{-1}\right)\end{array}$ \\
\hline Chloroform & $\mathrm{CHCl}_{3}$ & 17.8 & 3.1 & 5.7 & 18.9 & 0.001 & 0.4 & 1424 \\
\hline
\end{tabular}




\begin{tabular}{ccccccccc}
\hline DCE & $\mathrm{CH}_{2} \mathrm{ClCH}_{2} \mathrm{Cl}$ & 19.0 & 7.4 & 4.1 & 20.3 & 0.007 & 0.6 & 1724 \\
DMF & $\mathrm{HCON}\left(\mathrm{CH}_{3}\right)_{2}$ & 17.4 & 13.7 & 11.3 & 24.0 & 0.022 & 0.5 & 2220 \\
Toluene & $\mathrm{C}_{7} \mathrm{H}_{8}$ & 18.0 & 1.4 & 2.0 & 18.2 & $<0.001$ & 0.95 & 1349 \\
MCB & $\mathrm{C}_{6} \mathrm{H}_{5} \mathrm{Cl}$ & 19.0 & 4.3 & 2.0 & 19.4 & $<0.001$ & 0.9 & 1196 \\
o-DCB & $\mathrm{C}_{6} \mathrm{H}_{4} \mathrm{Cl}_{2}$ & 19.2 & 6.3 & 3.3 & 20.5 & 0.015 & 0.25 & 1650 \\
m-DCB & $\mathrm{C}_{6} \mathrm{H}_{4} \mathrm{Cl}_{2}$ & 19.7 & 5.1 & 2.7 & 20.1 & 0.004 & 0.4 & 1313 \\
TCB & $\mathrm{C}_{6} \mathrm{H}_{3} \mathrm{Cl}_{3}$ & 20.2 & 6.0 & 3.2 & 20.3 & 0.005 & 0.1 & 1658 \\
DBE & $\mathrm{CH}_{2} \mathrm{BrCH}_{2} \mathrm{Br}$ & 17.8 & 6.4 & 7.0 & 21.3 & 0.010 & 0.25 & 2593 \\
Nitromethane & $\mathrm{CH}_{3} \mathrm{NO}_{2}$ & 15.8 & 18.8 & 5.1 & 25.8 & $<0.001$ & 0.9 & 911 \\
NMP & $\mathrm{C}_{5} \mathrm{H}_{9} \mathrm{NO}$ & 18.0 & 12.3 & 7.2 & 22.8 & $0.020^{*}$ & $0.1 *$ & $3264 *$ \\
Acetonitrile & $\mathrm{CH}_{3} \mathrm{CN}$ & 15.3 & 18.0 & 6.1 & 24.2 & $<0.001$ & 0.98 & 641 \\
DMSO & $\left(\mathrm{CH}_{2}\right)_{2} \mathrm{SO}$ & 18.4 & 16.4 & 10.2 & 26.6 & 0.006 & 0.65 & 1785 \\
\hline
\end{tabular}

* Data from reference [12]. , Total Hildebrand Solubility Parameter, ${ }_{D},{ }_{P,}$, , three dimensional Hansen Solubility Parameters: ${ }_{D}$, Dispersion component, ${ }_{P}$, Polar component, ${ }_{H}$, Hydrogen bonding component. $={ }_{D}^{2}+{ }_{P}^{2}+{ }_{H}^{2}[11]$

\section{FIGURE CAPTIONS}

Figure 1 Fraction of the nanotube aggregates in MCB, TCB and DMF dispersions as a function of prepared concentration. Two samples of SWNTs/DMF dispersions with concentration of $0.0375 \mathrm{mg} / \mathrm{ml}$ (A) and $0.0067 \mathrm{mg} / \mathrm{ml}$ (B) and one sample of SWNTs/TCB dispersion at concentration of $0.00282 \mathrm{mg} / \mathrm{ml}$ (C) were studied by AFM. 
Figure 2 AFM images of SWNTs/DMF dispersions after centrifugation, (a) $0.0375 \mathrm{mg} / \mathrm{ml}$, (b) 0.0067 $\mathrm{mg} / \mathrm{ml}$.

Figure 3 Position of the employed solvents in Hansen parameter space, the size of the sphere indicates the ease of dispersion of SWNTs (dispersion limit) in the corresponding solvent. For the dispersion limit below $0.001 \mathrm{mg} / \mathrm{ml}, 0.0005 \mathrm{mg} / \mathrm{ml}$ is used to indicate the sphere size.

Figure 4 Absorption coefficients and Dispersion limits as a function of Hildebrand parameter ( ).

Figure 5 Absorption coefficients and Dispersion limits $v s$ (a) dispersion component $\left({ }_{D}\right)$, (b) polar component $\left({ }_{P}\right)$, and (c) hydrogen-bonding component $\left({ }_{H}\right)$.

Figure 6 AFM image of DMF dispersion at $~ 0.003 \mathrm{mg} / \mathrm{ml}$ precentrifugation.

Figure 7 Aggregation fractions of SWNTs in DMF at different sonication time (volume 5ml, without temperature control).

Figure $8 \mathrm{I}_{\mathrm{D}} / \mathrm{I}_{\mathrm{G}}{ }^{+}$ratio as a function of sonication time.

Figure 9 AFM images of SWNTs dispersed in TCB at $0.00282 \mathrm{mg} / \mathrm{ml}$. 


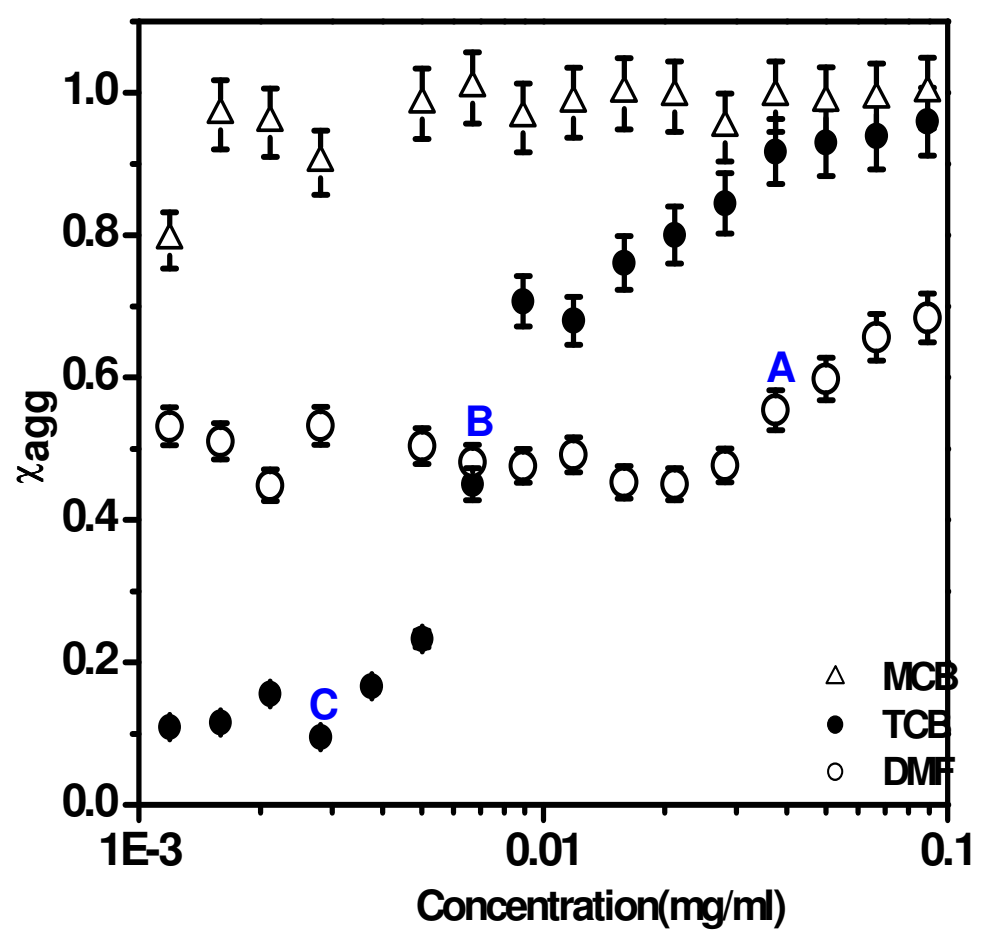

Figure 1
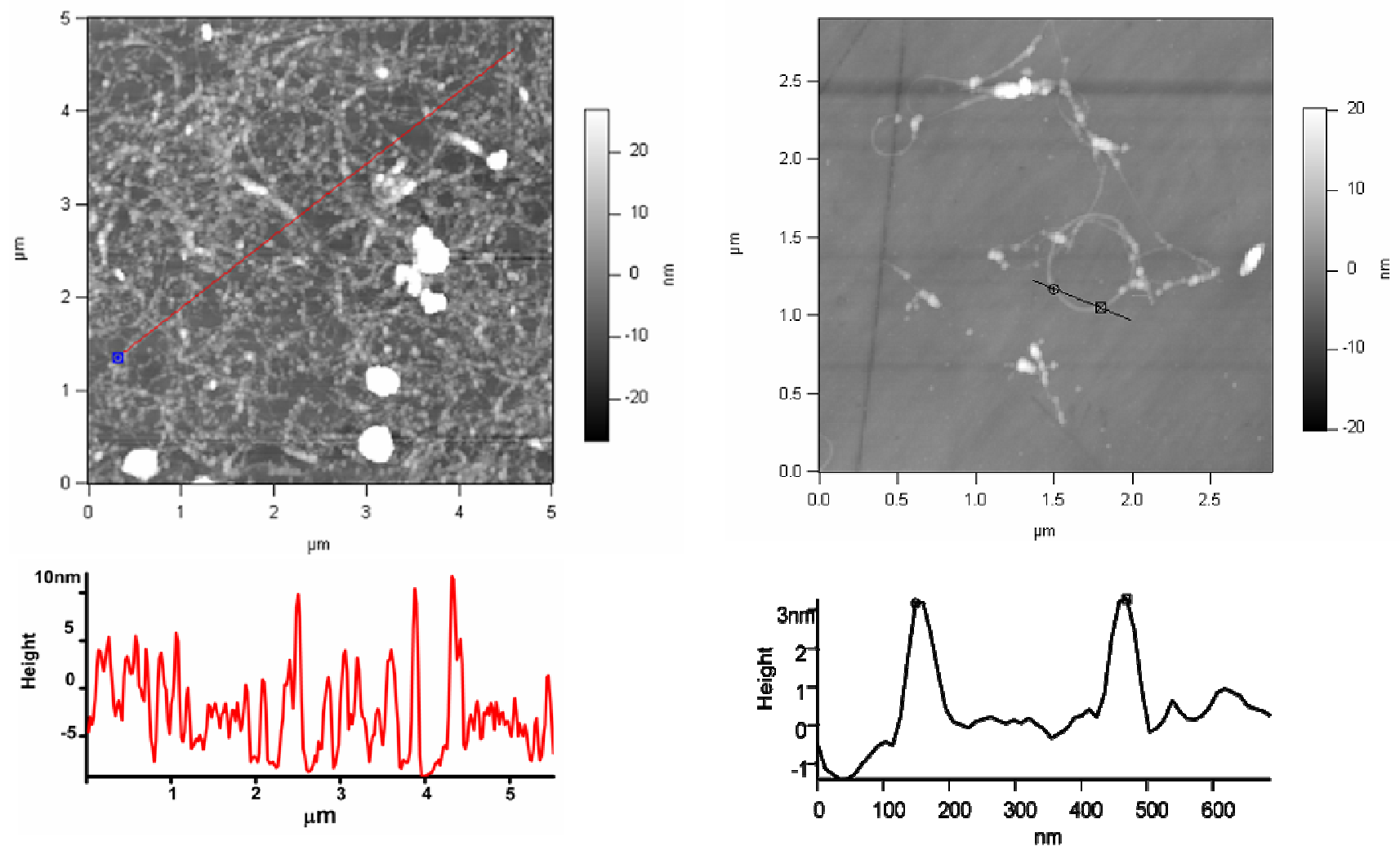

(a)

Figure 2

(b) 


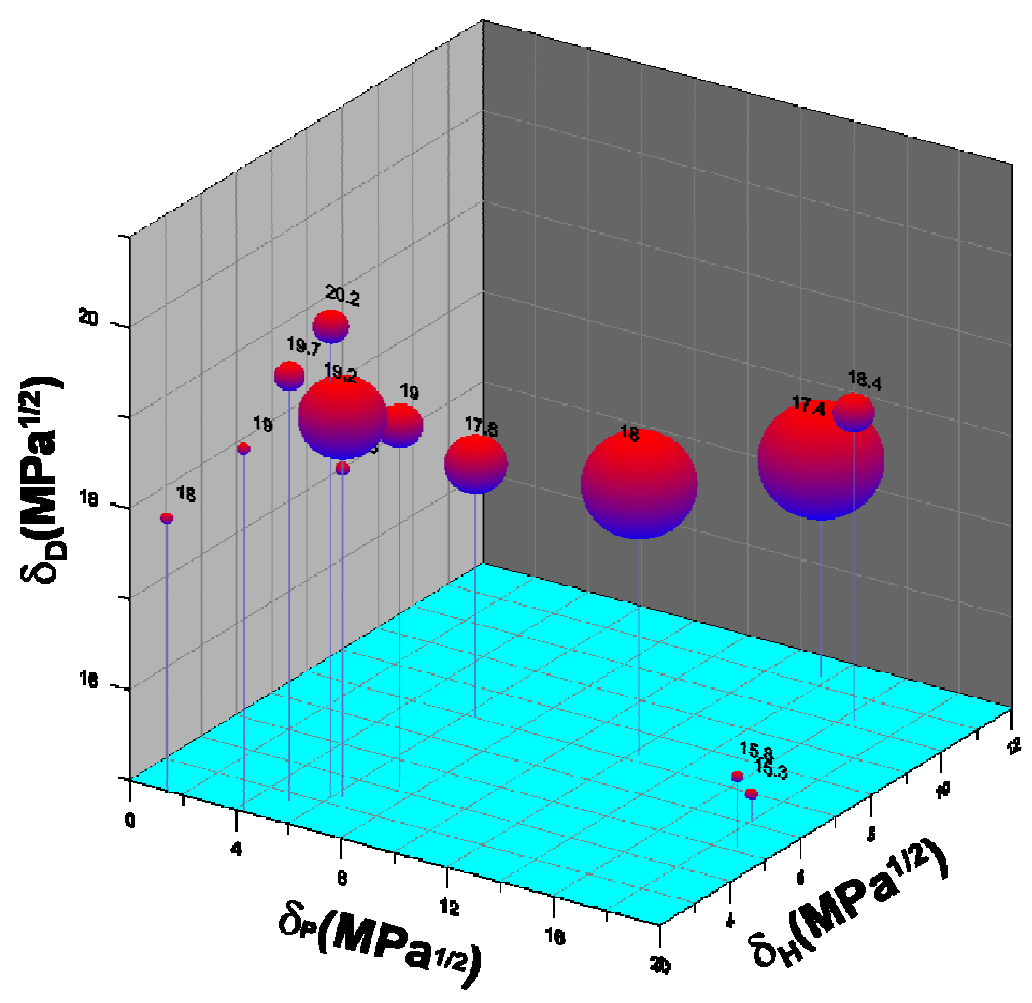

Figure 3

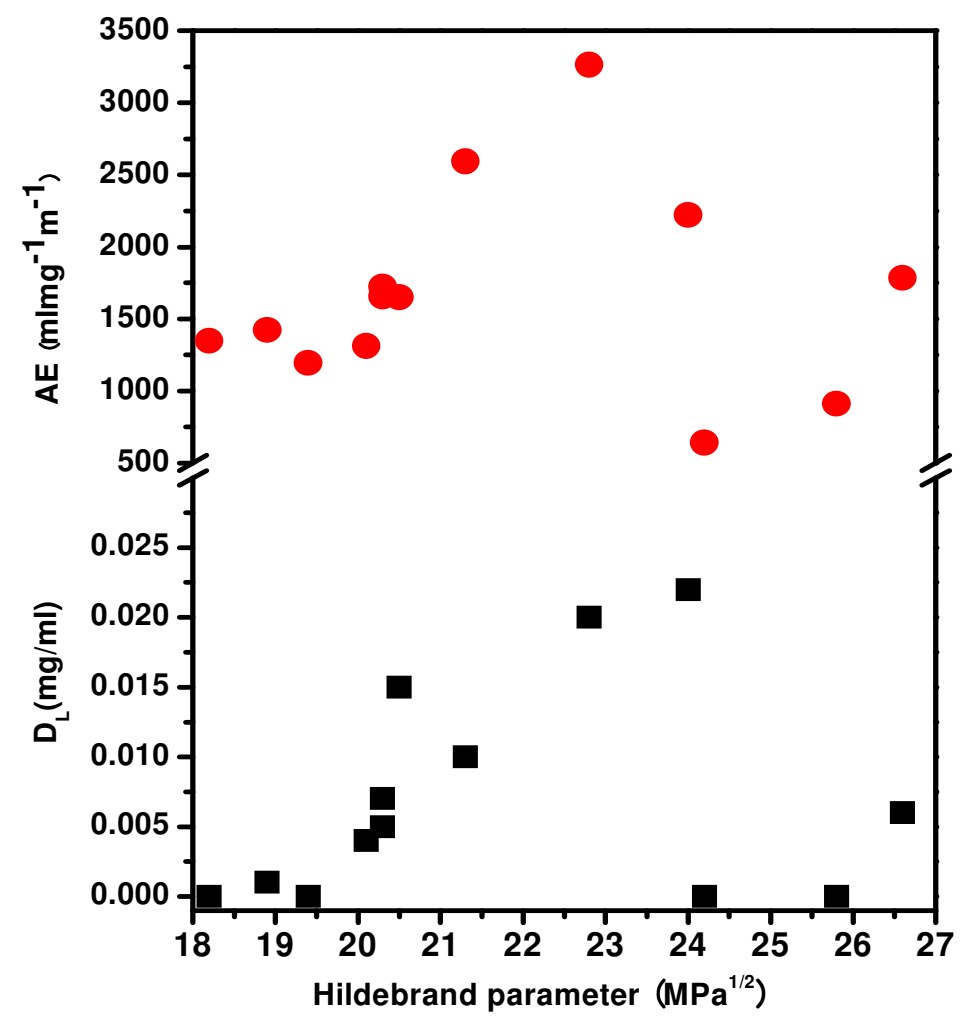

Figure 4 

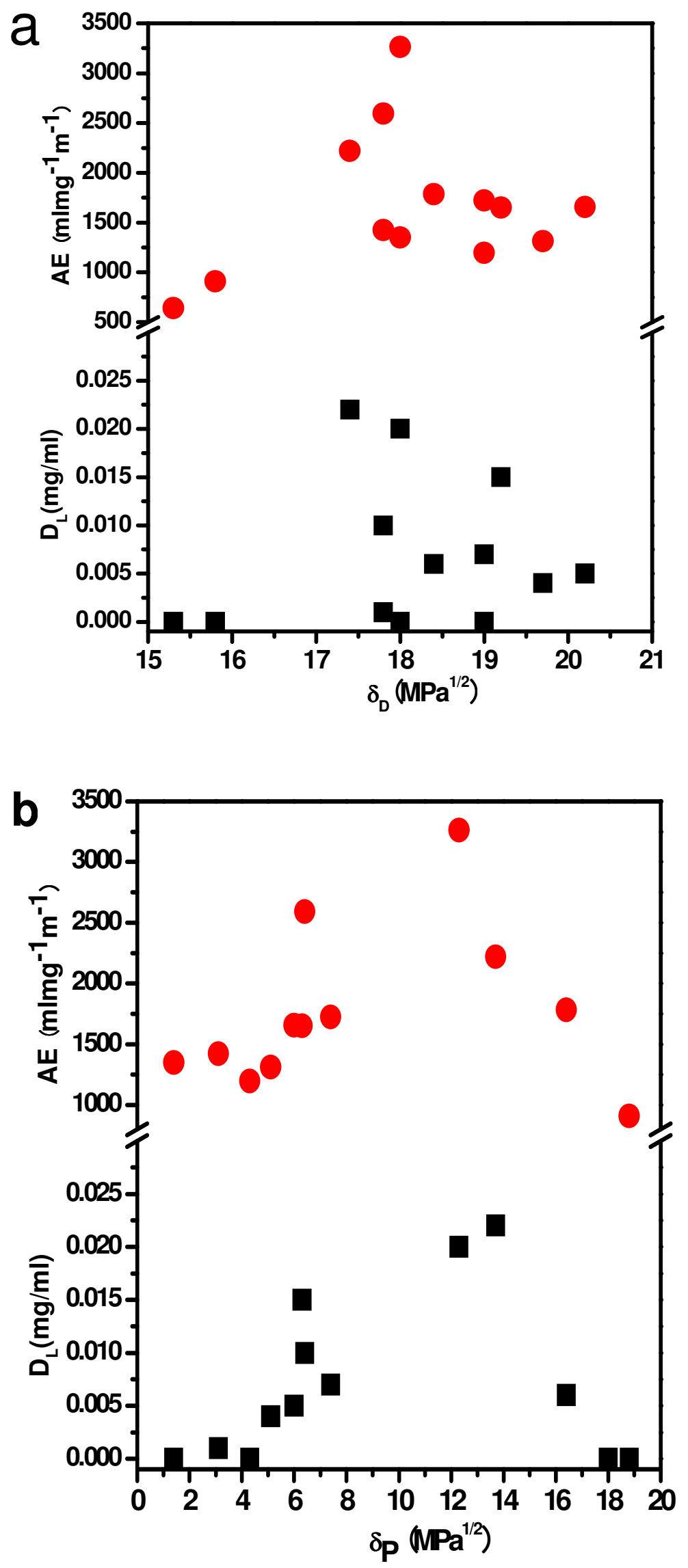


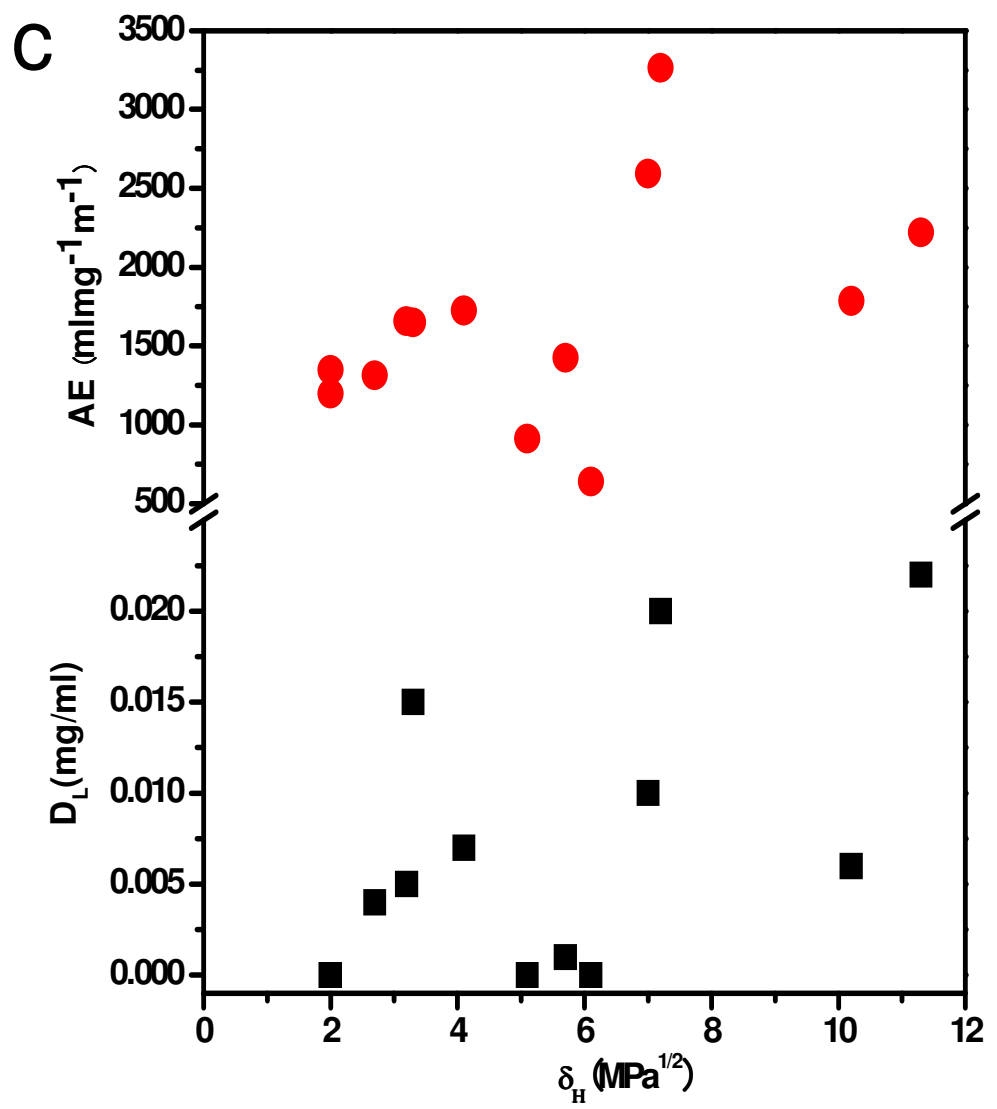

Figure 5
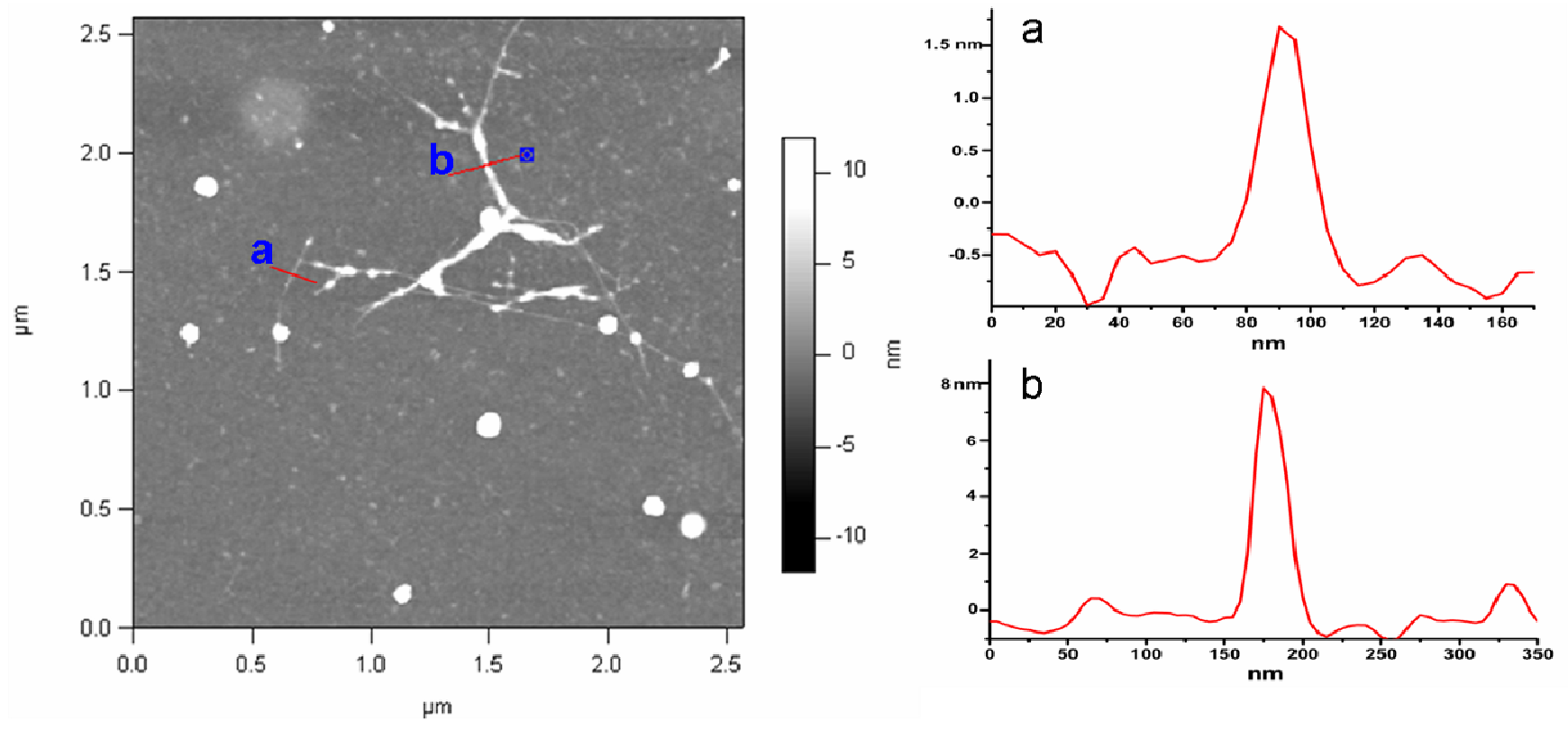

Figure 6 


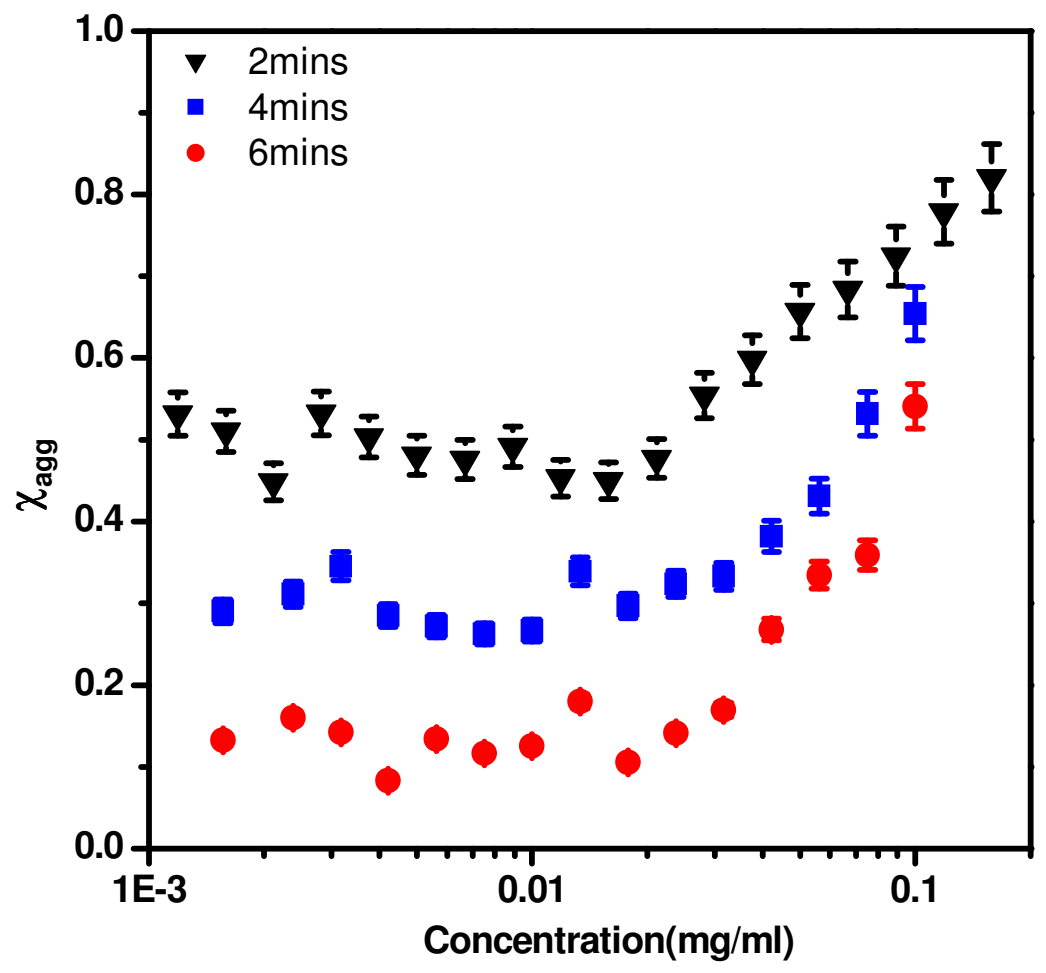

Figure 7

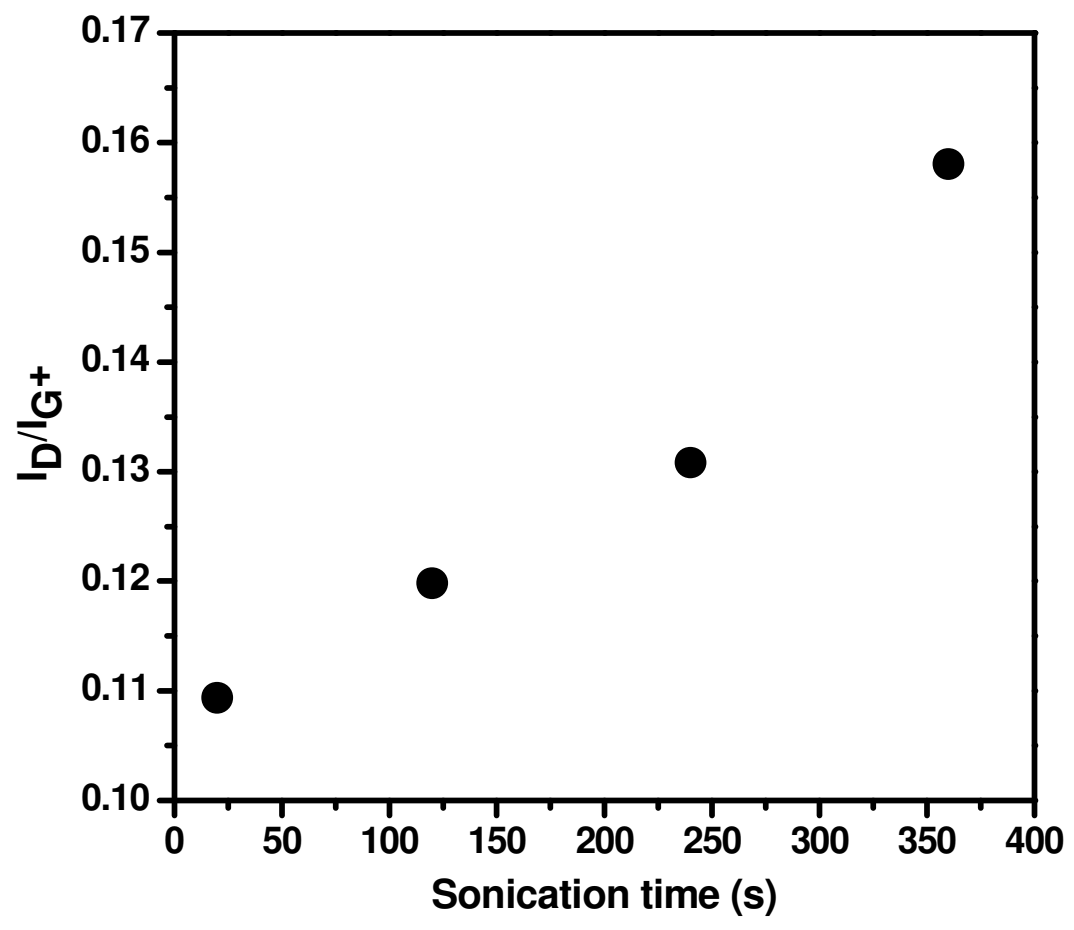

Figure 8 

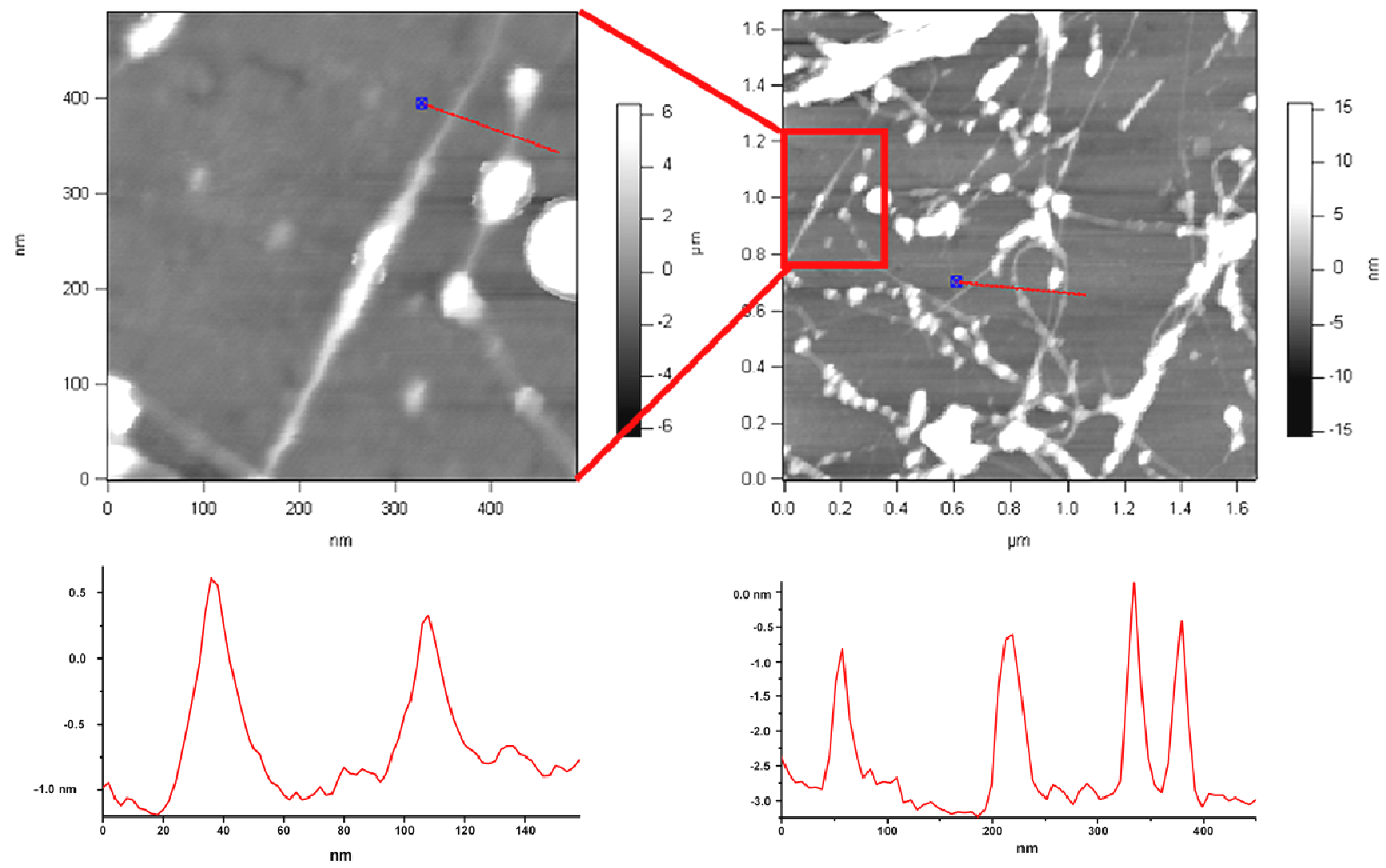

Figure 9 